\title{
Exact Computation of the Triangular-Lattice Ising Model with Eighteen Spins on a Side
}

\author{
Seung-Yeon Kim
}

\begin{abstract}
The Ising model, consisting of magnetic spins, is the most important system in understanding phase transitions and critical phenomena. For the first time, the exact integer values for the density of states of the triangular-lattice Ising model with eighteen spins on a side and free boundary conditions are evaluated. Also, the exact specific heats are obtained for the triangular-lattice Ising ferromagnet and antiferromagnet at the same time.
\end{abstract}

Index Terms-Exact computation, triangular-lattice Ising model, density of states.

\section{INTRODUCTION}

Phase transitions and critical phenomena are the most universal phenomena in nature. The Ising model, consisting of magnetic spins, is the simplest system showing phase transitions and critical phenomena at finite temperatures. The Ising model has played a central role in our understanding of phase transitions and critical phenomena [1]. Also, the Ising model explains the gas-liquid phase transitions accurately. Based on the Ising model, various theoretical methods such as mean-field theory, power-series expansion and analysis, renormalization group, and canonical transfer matrix have been developed to understand phase transitions and critical phenomena.

In particular, computer simulations have been the most popular method in studying phase transitions and critical phenomena due to the recent fast growth of computer hardware and software technologies. To investigate the phase transition and critical behavior of a given system as a continuous function of temperature, to obtain the partition function zeros showing most effectively phase transitions and critical phenomena, and to perform microcanonical analysis for phase transitions and critical phenomena, we need to calculate the density of states as a function of energy. The most computational methods to calculate the density of states yield the approximate density of state [2]-[13].

On the other hand, the microcanonical transfer matrix [14]-[39] is an exact computation method to calculate the exact integer values for the density of states. Until now, the exact integer values for the density of states of the Ising model on equilateral triangular lattice with free boundary conditions have been obtained up to fifteen spins on a side

Manuscript received November 26, 2014; revised May 12, 2015. This research was supported by Basic Science Research Program through the National Research Foundation of Korea (NRF) funded by the Ministry of Education (grant number NRF-2014R1A1A2056127).

Seung-Yeon Kim is with the School of Liberal Arts and Sciences, Korea National University of Transportation, Chungju 380-702, Republic of Korea (e-mail: sykimm@ut.ac.kr). (corresponding to $2^{120} \approx 1.3 \times 10^{36}$ states) [15]. In this work, for the first time, we evaluate the exact integer values for the density of states of the triangular-lattice Ising model with eighteen spins on a side (corresponding to $2^{171} \approx 3.0 \times 10^{51}$ states). It is very difficult task to classify all $2^{171}$ spin configurations according to their energy values.

Using the exact density of states of the triangular-lattice Ising model with eighteen spins on a side, we obtain much more accurately the specific heats of two different systems (the triangular-lattice Ising ferromagnet and antiferromagnet) at the same time. Based on the specific heats of the triangular-lattice Ising ferromagnet and antiferromagnet, we discuss the phase transitions and critical phenomena of these systems.

\section{ISING MODEL}

The Ising model on the equilateral triangular lattice [15] with eighteen spins on a side and free boundary conditions is defined by the Hamiltonian

$$
H=J \sum_{<i, j>}\left(1-\sigma_{i} \sigma_{j}\right)
$$

where $J$ is the coupling constant, $\langle i, j\rangle$ indicates a sum over all bonds between any nearest-neighbor spin pairs $\sigma_{i}$ and $\sigma_{j}$, and $\sigma_{i}= \pm 1$ ( 1 for upward magnetic spin and -1 for downward magnetic spin). On triangular lattice, each spin has the six nearest neighbor spins except for the spins on the boundary edges. The triangular-lattice Ising model with $L$ spins on a side has $N=L(L+1) / 2$ spins and $B=3(N-L)$ bonds. Therefore, there are $N=171$ spins and $B=459$ bonds for the equilateral triangular lattice with eighteen spins on a side $(L=18)$ and free boundary conditions.

Next, we define the density of states, $\Omega(E)$, with a given energy

$$
E=\sum_{<i, j>}\left(1-\sigma_{i} \sigma_{j}\right)
$$

where $E$ is integers between 0 and $4 B / 3=612$ for $L=18$. Then, the partition function of the triangular-lattice Ising model (a sum over all possible spin configurations)

$$
Z=\sum_{\{\sigma\}} \exp (-\beta H)
$$

where $\beta=1 / k T$ ( $k$ is the Boltzmann constant and $T$ is temperature), can be written as

$$
Z(T)=\sum_{E=0}^{612} \Omega(E) \exp (-\beta J E) .
$$

The partition function is the most important function in 
thermodynamics, statistical mechanics, and physical chemistry.

\section{DENSITY OF STATES}

The microcanonical transfer matrix [14]-[39], an exact computation method, is applied to calculate the exact integer values for the density of states of the Ising model on the equilateral triangular lattice with eighteen spins on a side ( $L=18, N=171, B=459)$. First, an array $\omega^{(1)}$, which is indexed by energy $E$ and the eighteen spin variables $\sigma_{i}^{(1)}(1 \leq i \leq 18)$ for the first row, is initialized with the seventeen horizontal bonds as

$$
\omega^{(1)}\left(E ; \sigma^{(1)}\right)=\delta\left(E+\sum_{n=1}^{17} \sigma_{n}^{(1)} \sigma_{n+1}^{(1)}-17\right),
$$

where $\delta$ is the Kronecker delta. Second, by introducing the seventeen spin variables $\sigma_{i}^{(2)}(1 \leq i \leq 17)$ for the second row and considering the thirty-four vertical bonds between the first and second rows, the array $\omega^{(1)}$ is modified into

$$
\varpi^{(2)}\left(E ; \sigma^{(2)}\right)=\omega^{(1)}\left(E+\sum_{n=1}^{17} \sigma_{n}^{(2)}\left(\sigma_{n}^{(1)}+\sigma_{n+1}^{(1)}\right)-34 ; \sigma^{(1)}\right) \text {. }
$$

After taking the sixteen horizontal bonds of the second-row spins, we obtain

$$
\omega^{(2)}\left(E ; \sigma^{(2)}\right)=\varpi^{(2)}\left(E+\sum_{n=1}^{16} \sigma_{n}^{(2)} \sigma_{n+1}^{(2)}-16 ; \sigma^{(2)}\right) .
$$

Next, for the third row, if we introduce the sixteen spin variables $\sigma_{i}^{(3)}(1 \leq i \leq 16)$ and consider the thirty-two vertical bonds, we have

$$
\varpi^{(3)}\left(E ; \sigma^{(3)}\right)=\omega^{(2)}\left(E+\sum_{n=1}^{16} \sigma_{n}^{(3)}\left(\sigma_{n}^{(2)}+\sigma_{n+1}^{(2)}\right)-32 ; \sigma^{(2)}\right) \text {. }
$$

Now, the fifteen horizontal bonds connecting the spins in the third row are taken into account by shifting the energy:

$$
\omega^{(3)}\left(E ; \sigma^{(3)}\right)=\varpi^{(3)}\left(E+\sum_{n=1}^{15} \sigma_{n}^{(3)} \sigma_{n+1}^{(3)}-15 ; \sigma^{(3)}\right) .
$$

After repeating these steps, the final spin $\sigma_{1}^{(18)}$ in the eighteenth row is introduced with the two vertical bonds such as

$$
\varpi^{(18)}\left(E ; \sigma^{(18)}\right)=\omega^{(17)}\left(E+\sigma_{1}^{(18)}\left(\sigma_{1}^{(17)}+\sigma_{2}^{(17)}\right)-2 ; \sigma^{(17)}\right) .
$$

Finally, the exact integer values for the density of states of the triangular-lattice Ising model with eighteen spins on a side is given by

$$
\Omega(E)=\sum_{\sigma} \varpi^{(18)}\left(E ; \sigma_{1}^{(18)}\right)
$$

as shown in Table I, II, and III. The sum over all densities of states is exactly equal to the number of all possible spin

\begin{tabular}{|c|c|}
\hline E & $\Omega(E)$ \\
\hline 0 & 2 \\
\hline 4 & 6 \\
\hline 8 & 120 \\
\hline 12 & 698 \\
\hline 16 & 4926 \\
\hline 20 & 32898 \\
\hline 24 & 183598 \\
\hline 28 & 1077360 \\
\hline 32 & 5732928 \\
\hline 36 & 29742908 \\
\hline 40 & 149995056 \\
\hline 44 & 725682150 \\
\hline 48 & 3441025398 \\
\hline 52 & 15850948398 \\
\hline 56 & 71378421714 \\
\hline 60 & 314842377450 \\
\hline 64 & 1360270736442 \\
\hline 68 & 5773198112370 \\
\hline 72 & 24079429379534 \\
\hline 76 & 98835422837142 \\
\hline 80 & 399631763275344 \\
\hline 84 & 1593020943584184 \\
\hline 88 & 6266017974032538 \\
\hline 92 & 24336615611053698 \\
\hline 96 & 93394373030137502 \\
\hline 100 & 354349069960204254 \\
\hline 104 & 1329908486113610586 \\
\hline 108 & 4939747629298345624 \\
\hline 112 & 18166324908534247938 \\
\hline 116 & 66172517125822650006 \\
\hline 120 & 238828273199602595280 \\
\hline 124 & 854324137189574254854 \\
\hline 128 & 3029724735335329863798 \\
\hline 132 & 10654321072330846416222 \\
\hline 136 & 37159871767171454952942 \\
\hline 140 & 128563609273897927138074 \\
\hline 144 & 441279588731867009146194 \\
\hline 148 & 1502816275339314440210718 \\
\hline 152 & 5078404212126656898914814 \\
\hline 156 & 17029451373598977602838314 \\
\hline 160 & 56668176518722621470880116 \\
\hline 164 & 187131754821406286835976524 \\
\hline 168 & 613227992362498144545682982 \\
\hline 172 & 1994123411279023994947380294 \\
\hline 176 & 6434584028140782226639532112 \\
\hline 180 & 20601752121939915250005359616 \\
\hline 184 & 65444705313464469115525800978 \\
\hline 188 & 206251950660318896085506890758 \\
\hline 192 & 644817488924589508544194155446 \\
\hline 196 & 1999625296622038907636275808550 \\
\hline 200 & 6150176507800464864457145926374 \\
\hline 204 & 18758764707707840199874164120552 \\
\hline 208 & 56734322502598178279959938358008 \\
\hline 212 & 170120524893135900490975462542828 \\
\hline 216 & 505682708225017649369928170719504 \\
\hline 220 & 1489870977644177452447531651457436 \\
\hline 224 & 4350151776353064838774025656713900 \\
\hline 228 & 12585743860761393660220709604901732 \\
\hline 232 & 36074742254773441282921967499712296 \\
\hline 236 & 102425068949841268490650936689064146 \\
\hline 240 & 288014606367086068382310243413145754 \\
\hline 244 & 801959230924593393823151339228959818 \\
\hline 248 & 2210760545258121573655221760353521826 \\
\hline
\end{tabular}
configurations:

$$
\sum_{E} \Omega(E)=2^{171} \approx 3.0 \times 10^{51}
$$

TABLE I: EXACT INTEGER VALUES FOR THE DENSITY OF STATES $\Omega(E)$ OF THE ISING MODEL ON THE EQUILATERAL TRIANGULAR LATTICE WITH EIGHTEEN SPINS ON A SiDE AND FREE BOUNDARY CONDITIONS, AS A FUNCTION OF ENERGY $E(=0 \sim 248)$

The ferromagnetic $(J>0)$ ground states correspond to 


$$
\Omega(E=0)=2,
$$

and the antiferromagnetic $(J<0)$ ground states are quite degenerate such as

$$
\Omega(E=612)=23665003296449525435806996826,
$$

corresponding approximately to $2.4 \times 10^{28}$.

The largest density of states is

$$
\begin{aligned}
\Omega(E=460)= & 223344742615528798625291061965 \\
& 299440306354840893412,
\end{aligned}
$$

corresponding approximately to $2.2 \times 10^{50}$. This kind of a large integer number is stored in a computer by using a positional numeral system with a radix (or base) of $2^{31}$ such as

$$
\Omega(E=460)=\sum_{j} P_{j}\left(2^{31}\right)^{j-1}
$$

Here, we need the six $P_{j}$ 's as follows:

$$
\begin{gathered}
P_{1}=143620068, \\
P_{2}=1321816796, \\
P_{3}=1227133195, \\
P_{4}=1023651300, \\
P_{5}=424290496,
\end{gathered}
$$

and

$$
P_{6}=4890 \text {. }
$$

It should be noted that the exact integer values for the density of states of the Ising model on the equilateral triangular lattice with eighteen spins on a side and free boundary conditions are obtained for the first time. Even the approximate values for the density of states of the triangular-lattice Ising model with eighteen spins on a side and free boundary conditions have never been calculated by using other non-exact methods.

\section{Exact Specific Heats}

Given the exact integer values for the density of states $\Omega(E)$, the free energy $F$ is exactly given by

$$
F=-k T \ln Z(T) \text {. }
$$

From the exact free energy, the exact thermodynamic functions can be obtained. For example, the exact specific heat can be expressed as [32], [37]

$$
C(T)=\left(N k T^{2}\right)^{-1} \frac{\partial^{2}}{\partial \beta^{2}} \ln Z(T) .
$$

It should be noted that the exact specific heats of two different systems (the ferromagnet and the antiferromagnet)

\begin{tabular}{|c|c|}
\hline E & $\Omega(E)$ \\
\hline 252 & 6032574296702207567992159617631054388 \\
\hline 256 & 16291195531867254409681753011959573286 \\
\hline 260 & 43531947414286528716741831764080745418 \\
\hline 264 & 115075126573566581358408907157488958812 \\
\hline 268 & 300873678653252315209962248506197217320 \\
\hline 272 & 777902547118917508886357885597026371366 \\
\hline 276 & 1988433439781483966312131893603101542126 \\
\hline 280 & 5023958673368655601048981585747322922204 \\
\hline 284 & 12543885244867446025652519130032502285132 \\
\hline 288 & 30943435281169248372287421652274949167448 \\
\hline 292 & 75396697989825485392612164879262241525340 \\
\hline 296 & 181416915513496537802958960691994207820984 \\
\hline 300 & 430959549961245438057562394617210011264808 \\
\hline 304 & 1010455910177551377990340814506184069953140 \\
\hline 308 & 2337794510734717746244322204432816224734456 \\
\hline 312 & 5335638573482537573490477967589333546397300 \\
\hline 316 & 12009846987882627333999323765431938145155756 \\
\hline 320 & 26652393902684429250696803548595613647326734 \\
\hline 324 & 58298310736110255123305274354978237303067770 \\
\hline 328 & 125651170264436331856921237358213453896069668 \\
\hline 332 & 266768220748538447476589754247011255114467706 \\
\hline 336 & 557726120368120620395722344793580882465983536 \\
\hline 340 & 1147851147704756884493869558888840041533574048 \\
\hline 344 & 2324780929925745325235296380495955520750686530 \\
\hline 348 & 4631910578468230284770764119614881772222106046 \\
\hline 352 & 9075387362796883330336224354705276013549719348 \\
\hline 356 & 17479838923327154950510934380492643075364900888 \\
\hline 360 & 33083560464058716419199680375817998937570681962 \\
\hline 364 & 61506299526417504864955335620459187482958031548 \\
\hline 368 & 112275207284978890115800573591860818881023776790 \\
\hline 372 & 201151604933687224887819792212492026300496077482 \\
\hline 376 & 353549914805816553550110672952201751485673345844 \\
\hline 380 & 609356184799887613362709199930535233554098666606 \\
\hline 384 & 1029398830001625559753762008003729222640666522202 \\
\hline 388 & 1703646342247042457834241306547706659577566228218 \\
\hline 392 & 2760845787272406555573996114171202185297260231814 \\
\hline 396 & 4378719827587143093460219433647641846027105118498 \\
\hline 400 & 6792992427163107607330591388598583409311604038806 \\
\hline 404 & 10302472823119506572551114792442523831590149014978 \\
\hline 408 & 15266370194980358625705190888815790266438642426302 \\
\hline 412 & 22089262612143002704248051819439686486356276466066 \\
\hline 416 & 31189169632766629985795474696554089831603362694564 \\
\hline 420 & 42945512870251241349677149761961196285738164376340 \\
\hline 424 & 57626836356124675863137961179245217030105494120710 \\
\hline 428 & 75303113020939996316545143187513130695899814814958 \\
\hline 432 & 95753806349040845154443893768987044017052089168192 \\
\hline 436 & 118389329416043473011419930462233300764094757778936 \\
\hline 440 & 142208141171905046740916723511813585532991827339074 \\
\hline 444 & 165812058367006765384057007646193071136749750987574 \\
\hline 448 & 187496418825096013726516930528609480748581983635754 \\
\hline 452 & 205418817021640234882736178728743807730007752178450 \\
\hline 456 & 217831755706261800880699734153843652400701430461078 \\
\hline 460 & 223344742615528798625291061965299440306354840893412 \\
\hline 464 & 221166015404198799821872320331558576084831981659624 \\
\hline 468 & 211269309152492812119488374854525701462767985296124 \\
\hline 472 & 194440864822807089868319235365666649412871411180940 \\
\hline 476 & 172185929905909902442436348164816469818599994947408 \\
\hline 480 & 146506912841695542187622155503529962943797769036172 \\
\hline 484 & 119597709009310355684848476855733332051653835280332 \\
\hline 488 & 93520159958647237984995369509626383559951598328372 \\
\hline 492 & 69931766814162508421556977727470656380144332831802 \\
\hline 496 & 49917393007869262174557191391305210208311870740686 \\
\hline 500 & 33947391382667604774400754087373481647747354286902 \\
\hline
\end{tabular}
can be obtained at the same time in this work.

Fig. 1 shows the exact specific heat of the Ising ferromagnet $(J>0)$ on the equilateral triangular lattice with eighteen spins on a side and free boundary conditions. The specific heat shows the sharp peak at $\mathrm{T}=3.232 \mathrm{~J} / \mathrm{k}$, signaling the phase transition between the low-temperature ferromagnetic phase and the high-temperature paramagnetic phase.

TABLE II: EXACt INTEGER VALUES For the Density of States $\Omega(E)$ OF THE ISING MODEL ON THE EQUILATERAL TRIANGULAR LATTICE WITH EIGHTEEN SPINS ON A SIDE AND FREE BOUNDARY CONDITIONS, AS A FUNCTION OF ENERGY E $(=252 \sim 500)$ 
TABLE III: EXACT INTEGER VALUES FOR THE DENSITY OF STATES $\Omega(E)$ OF THE ISING MODEL ON THE EQUILATERAL TRIANGULAR LATTICE WITH EIGHTEEN SPINS ON A SiDE AND FREE BOUNDARY CONDITIONS, AS A FUNCTION OF ENERGY E (=504 612)

\begin{tabular}{ll}
\hline \hline$E$ & $\Omega(E)$ \\
\hline 504 & 21950781726445057354293572646890974133910427062586 \\
508 & 13465804322590765693109900843417171490205709454840 \\
512 & 7818704538801627208348282883093631189281721542472 \\
516 & 4286118920863464319012351239079820313385928372184 \\
520 & 2212305652992360430441136002143791362484968188504 \\
524 & 1072041653307650893550563631998246073083876491714 \\
528 & 486181006450383850841607109660327484773133664052 \\
532 & 205649417094675483217121122612034006852750305776 \\
536 & 80835122735378429057361360565289896153212053658 \\
540 & 29408820840369626431217743962720078903176877928 \\
544 & 9859751964338024711704821054735749328800880912 \\
548 & 3031755345218311633082572397197569224058917256 \\
552 & 850526355685300635904139272904828377861799116 \\
556 & 216439491775713945247246780347735395063388510 \\
560 & 49642320030960240892240905384932124041386758 \\
564 & 10188582054939574080136950772359492660736290 \\
568 & 1856076608608365806023004991979821849783174 \\
572 & 297351124582870094358418260738393802564494 \\
576 & 41444399841439401286605731668575495441304 \\
580 & 4962271488410140540704733439860177529052 \\
584 & 502673946829119573918563783000154607602 \\
588 & 42275793284432184726316549690456143940 \\
592 & 2881565478570894451055004821430792480 \\
596 & 154143255292560847456301000415328128 \\
600 & 6182853767838614190348673645213992 \\
604 & 173315951796170858726377113576180 \\
608 & 2994826377002713783475651283510 \\
612 & 23665003296449525435806996826 \\
\hline \hline
\end{tabular}

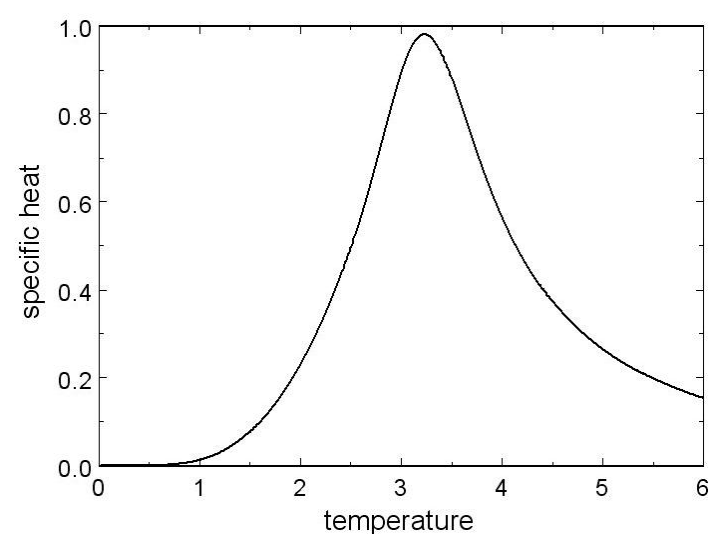

Fig. 1. Exact specific heat (in units of the Boltzmann constant $k$ ) per volume as a function of temperature (in units of $J / k$ ) for the triangular-lattice Ising ferromagnet $(J>0)$ with eighteen spins on a side.

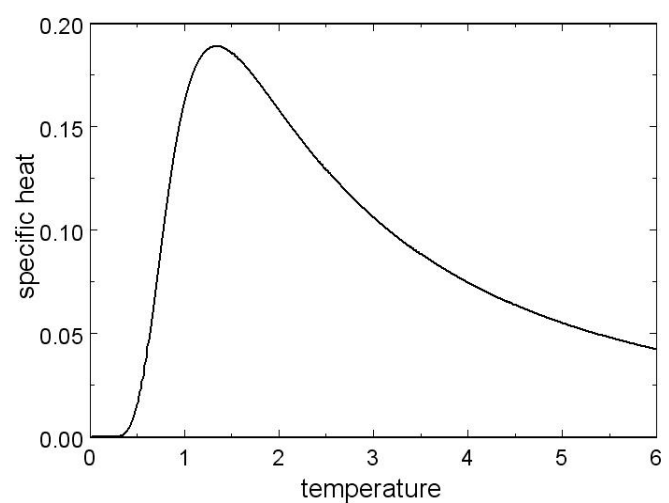

Fig. 2. Exact specific heat (in units of $k$ ) per volume as a function of temperature (in units of $|J| / k$ ) for the triangular-lattice Ising antiferromagnet $(J<0)$ with eighteen spins on a side.
Fig. 2 shows the exact specific heat of the Ising antiferromagnet $(J<0)$ on the equilateral triangular lattice with eighteen spins on a side and free boundary conditions. The specific heat shows a peak at $T=1.340|J| / k$. But the peak of the triangular-lattice Ising antiferromagnet is not sharp, compared to the peak of the triangular-lattice Ising ferromagnet. Rather, the peak for the specific heat of the triangular-lattice Ising antiferromagnet resembles the Schottky-anomaly peak for the specific heat of the one-dimensional Ising model [40].

\section{CONCLUSION}

For the first time, we have evaluated the exact integer values for the density of states of the Ising model on the equilateral triangular lattice with eighteen spins on a side and free boundary conditions, by classifying all $2^{171} \approx 3.0 \times 10^{51}$ spin configurations according to their energy values. Using the exact density of states of the triangular-lattice Ising model with eighteen spins on a side, we have obtained much more accurately the specific heats of two different systems (the triangular-lattice Ising ferromagnet and antiferromagnet) at the same time. Based on the specific heats of the triangular-lattice Ising ferromagnet and antiferromagnet, we have investigated the phase transitions and critical phenomena of these systems. The specific heat of the triangular-lattice Ising ferromagnet has shown a sharp peak, signaling the phase transition between the low-temperature ferromagnetic phase and the high-temperature paramagnetic phase. On the other hand, the specific heat of the triangular-lattice Ising antiferromagnet has shown the Schottky-anomaly peak.

\section{REFERENCES}

[1] C. Domb, The Critical Point, London, U.K.: Taylor and Francis, 1996.

[2] G. Bhanot, R. Salvador, S. Black, P. Carter, and R. Toral, "Accurate estimate of $v$ for the three-dimensional Ising model from a numerical measurement of its partition function," Physical Review Letters, vol. 59, pp. 803-806, 1987.

[3] B. A. Berg and T. Neuhaus, "Multicanonical ensemble: A new approach to simulate first-order phase transitions," Physical Review Letters, vol. 68, pp. 9-12, 1992.

[4] J. Lee, "New Monte Carlo algorithm: Entropic sampling," Physical Review Letters, vol. 71, pp. 211-214, 1993.

[5] F. Wang and D. P. Landau, "Multiple-range random walk algorithm to calculate the density of states," Physical Review Letters, vol. 86, pp. 2050-2053, 2001

[6] C.-O. Hwang, S.-Y. Kim, D. Kang, and J. M. Kim, "Ising antiferromagnets in a nonzero uniform magnetic field," Journal of Statistical Mechanics, vol. 7, pp. 1-8, 2007.

[7] S.-Y. Kim, C.-O. Hwang, and J. M. Kim, "Partition function zeros of the antiferromagnetic Ising model on triangular lattice in the complex temperature plane for nonzero magnetic field," Nuclear Physics B, vol. 805, pp. 441-450, 2008.

[8] C.-O. Hwang and S.-Y. Kim, "Yang-Lee zeros of triangular Ising antiferromagnets," Physica A, vol. 389, pp. 5650-5654, 2010.

[9] J. H. Lee, H. S. Song, J. M. Kim, and S.-Y. Kim, "Study of a square-lattice Ising superantiferromagnet using the Wang-Landau algorithm and partition function zeros," Journal of Statistical Mechanics, vol. 10, pp. 1-9, 2010.

[10] S.-Y. Kim, "Evaluation of Wang-Landau Monte Carlo simulations," International Proceedings of Computer Science and Information Technology, vol. 22, pp. 125-129, 2012.

[11] S.-Y. Kim, "Evaluation of an efficient Monte Carlo algorithm to calculate the density of states," International Journal of Machine Learning and Computing, vol. 2, pp. 144-149, 2012. 
[12] S.-Y. Kim and W. Kwak, "Study of the antiferromagnetic Blume-Capel model by using the partition function zeros in the complex temperature plane," Journal of the Korean Physical Society, vol. 65, pp. 436-440, 2014.

[13] C.-O. Hwang and S.-Y. Kim, "Field-induced Kosterlitz-Thouless transition in critical triangular-lattice antiferromagnets," Monte Carlo Methods and Applications, vol. 20, pp. 217-221, 2014.

[14] G. Bhanot, "A numerical method to compute exactly the partition function with applications to $Z(n)$ theories in two dimensions," Journal of Statistical Physics, vol. 60, pp. 55-75, 1990.

[15] B. Stosic, S. Milosevic, and M. E. Stanley, "Exact results for the two-dimensional Ising model in a magnetic field: Tests of finite-size scaling theory," Physical Review B, vol. 41, pp. 11466-11478, 1990.

[16] R. J. Creswick, "Transfer matrix for the restricted canonical and microcanonical ensembles," Physical Review E, vol. 52, pp. R5735-R5738, 1995.

[17] R. J. Creswick and S.-Y. Kim, "Finite-size scaling of the density of zeros of the partition function in first- and second-order phase transitions," Physical Review E, vol. 56, pp. 2418-2422, 1997.

[18] S.-Y. Kim and R. J. Creswick, "Yang-Lee zeros of the $Q$-state Potts model in the complex magnetic field plane," Physical Review Letters, vol. 81, pp. 2000-2003, 1998.

[19] S.-Y. Kim and R. J. Creswick, "Fisher zeros of the $Q$-state Potts model in the complex temperature plane for nonzero external magnetic field,' Physical Review E, vol. 58, pp. 7006-7012, 1998.

[20] R. J. Creswick and S.-Y. Kim, "Microcanonical transfer matrix study of the $Q$-state Potts model," Computer Physics Communications, vol. 121 , pp. 26-29, 1999.

[21] S.-Y. Kim and R. J. Creswick, "Exact results for the zeros of the partition function of the Potts model on finite lattices," Physica A, vol 281, pp. 252-261, 2000.

[22] S.-Y. Kim and R. J. Creswick, "Density of states, Potts zeros, and Fisher zeros of the $Q$-state Potts model for continuous $Q$," Physical Review E, vol. 63, article: 066107, pp. 1-12, 2001.

[23] S.-Y. Kim, "Partition function zeros of the Q-state Potts model on the simple-cubic lattice," Nuclear Physics B, vol. 637, pp. 409-426, 2002.

[24] S.-Y. Kim, "Density of the Fisher zeros for the three-state and four-state Potts models," Physical Review E, vol. 70, pp. 1-5, 2004.

[25] S.-Y. Kim, "Yang-Lee zeros of the antiferromagnetic Ising model," Physical Review Letters, vol. 93, pp. 1-4, 2004.

[26] S.-Y. Kim, "Fisher zeros of the Ising antiferromagnet in an arbitrary nonzero magnetic field plane," Physical Review E, vol. 71, pp. 1-4, 2005 .

[27] S.-Y. Kim, "Density of Yang-Lee zeros and Yang-Lee edge singularity for the antiferromagnetic Ising model," Nuclear Physics B, vol. 705, pp. 504-520, 2005 .

[28] S.-Y. Kim, "Honeycomb-lattice antiferromagnetic Ising model in a magnetic field," Physics Letters A, vol. 358, pp. 245-250, 2006.
[29] S.-Y. Kim, "Density of Yang-Lee zeros for the Ising ferromagnet," Physical Review E, vol. 74, pp. 1-7, 2006.

[30] M. E. Monroe and S.-Y. Kim, "Phase diagram and critical exponent $v$ for nearest-neighbor and next-nearest-neighbor interaction Ising model," Physical Review E, vol. 76, article: 021123, pp. 1-5, 2007.

[31] S.-Y. Kim, "Ground-state entropy of the square-lattice $Q$-state Potts antiferromagnet," Journal of the Korean Physical Society, vol. 52, pp 551-556, 2008.

[32] S.-Y. Kim, "Specific heat of the square-lattice Ising antiferromagnet in a magnetic field," Journal of Physical Studies, vol. 13, pp. 1-3, 2009.

[33] S.-Y. Kim, "Partition function zeros of the square-lattice Ising model with nearest- and next-nearest-neighbor interactions," Physical Review E, vol. 81, pp. 1-7, 2010.

[34] S.-Y. Kim, "Partition function zeros of the honeycomb-lattice Ising antiferromagnet in the complex magnetic-field plane," Physical Review E, vol. 82, pp. 1-7, 2010

[35] S.-Y. Kim, "Honeycomb-lattice Ising model in a nonzero magnetic field: Low-temperature series analysis and partition function zeros," Journal of the Korean Physical Society, vol. 56, pp. 1051-1054, 2010.

[36] S.-Y. Kim, "Yang-Lee edge singularity of the square-lattice Ising ferromagnet," Journal of the Korean Physical Society, vol. 59, pp. 2205-2208, 2011.

[37] S.-Y. Kim, "Specific heat and partition function zeros of the three-state Potts model," Journal of the Korean Physical Society, vol. 59, pp. 2980-2983, 2011.

[38] S.-Y. Kim, "Ising antiferromagnets on honeycomb and square lattices in the critical magnetic field," Journal of the Korean Physical Society, vol. 61, pp. 1950-1955, 2012.

[39] S.-Y. Kim, "Exact partition functions of the Ising model on $L \times L$ square lattices with free boundary conditions up to $L=22$," Journal of the Korean Physical Society, vol. 62, pp. 214-219, 2013.

[40] S.-Y. Kim, "Generalized Schottky anomaly," Journal of the Korean Physical Society, vol. 65, pp. 970-972, 2014.

Seung-Yeon Kim received the B.Sc. and M.Sc. degrees in physics from Yonsei University, Seoul, Republic of Korea, in 1990 and 1992, respectively. He received the Ph.D. degree in physics from University of South Carolina, Columbia, South Carolina, USA in 2000.

As a postdoctoral research fellow and a professor, he worked at Princeton University, New Jersey, USA, Korea Institute for Advanced Study, Seoul, Korea, and Soongsil University, Seoul, Korea before joining Korea National University of Transportation in 2006. Currently, he is a full professor at Korea National University of Transportation. His research interests are mathematical physics, computational physics, computational biology, and bioinformatics. 